\title{
Some Generalizations of Integral Inequalities of Hermite-Hadamard Type for n-Time Differentiable Functions
}

\author{
Tian-Yu Zhang', Bai-Ni Guo ${ }^{2, *}$ \\ ${ }^{1}$ College of Mathematics, Inner Mongolia University for Nationalities, Tongliao City, Inner Mongolia Autonomous Region, China \\ ${ }^{2}$ School of Mathematics and Informatics, Henan Polytechnic University, Jiaozuo City, Henan Province, China \\ *Corresponding author: bai.ni.guo@hotmail.com
}

Received March 02, 2015; Revised April 10, 2015; Accepted April 17, 2015

\begin{abstract}
In the paper, by establishing two integral identities and Hölder integral inequality, the authors generalize some integral inequalities of Hermite-Hadamard type for n-time differentiable functions on a closed interval.
\end{abstract}

Keywords: generalization, Hermite-Hadamard integral inequality, differentiable function, Hölder integral inequality

Cite This Article: Tian-Yu Zhang, and Bai-Ni Guo, "Some Generalizations of Integral Inequalities of Hermite-Hadamard Type for n-Time Differentiable Functions." Turkish Journal of Analysis and Number Theory, vol. 3, no. 2 (2015): 43-48. doi: 10.12691/tjant-3-2-2.

\section{Introduction}

Let $f(x)$ be a convex function on $[a ; b]$, the famous Hermite-Hadamard integral inequality may be expressed as

$$
\begin{aligned}
& 0 \leq \int_{a}^{b} f(t) d t-(b-a) f\left(\frac{a+b}{2}\right) \\
& \leq(b-a) \frac{f(a)+f(b)}{2}-\int_{a}^{b} f(t) d t .
\end{aligned}
$$

It is well known that Hermite-Hadamard integral inequality is an important cornerstone in mathematical analysis and optimization. There has been a growing literature considering its refinements and interpolations. For more information, please refer to the monographs $[3,4]$, the newly published papers [1,7], and plenty of references therein.

The following theorems are some refinements and generalizations of inequalities in (1.1).

Theorem 1.1 ([2] and [[5], Theorem A]). Let $f:[a, b] \in \mathbb{R} \rightarrow \mathbb{R}$ be a twice differentiable mapping and suppose that $\gamma \leq f^{\prime \prime}(t) \leq \Gamma$ for all $t \in(a, b)$. Then we have

$$
\frac{\gamma(b-a)^{2}}{24} \leq \frac{1}{b-a} \int_{a}^{b} f(t) d t-f\left(\frac{a+b}{2}\right) \leq \frac{\Gamma(b-a)^{2}}{24}
$$

and

$$
\frac{\gamma(b-a)^{2}}{12} \leq \frac{f(a)+f(b)}{2}-\frac{1}{b-a} \int_{a}^{b} f(t) d t \leq \frac{\Gamma(b-a)^{2}}{12} .
$$

Theorem 1.2 ([6] and [[5], Theorem B]). Let $f:[a, b] \in \mathbb{R} \rightarrow \mathbb{R}$ be a twice differentiable mapping and suppose that $\gamma \leq f^{\prime \prime}(t) \leq \Gamma$ for all $t \in(a, b)$, then

$$
\begin{aligned}
& \frac{3 S_{2}-2 \Gamma}{24}(b-a)^{2} \\
\leq & \frac{1}{b-a} \int_{a}^{b} f(t) d t-f\left(\frac{a+b}{2}\right) \\
\leq & \frac{3 S_{2}-2 \gamma}{24}(b-a)^{2}
\end{aligned}
$$

and

$$
\begin{aligned}
& \frac{3 S_{2}-2 \Gamma}{24}(b-a)^{2} \\
\leq & \frac{f(a)+f(b)}{2}-\frac{1}{b-a} \int_{a}^{b} f(t) d t \\
\leq & \frac{3 S_{2}-2 \gamma}{24}(b-a)^{2},
\end{aligned}
$$

where

$$
S_{n}=\frac{f^{(n-1)}(b)-f^{(n-1)}(a)}{b-a}, n \in \mathbb{N} .
$$

The above two theorems were further generalized by the following theorems.

Theorem 1.3 ([[5], Theorem 1]). Let $f(t)$ be n-time differentiable on the closed interval $[a, b]$ such that $\gamma \leq f^{(n)}(t) \leq \Gamma$ for $t \in[a, b]$ and $n \in \mathbb{N}$. Further, let $u \in[a, b]$ be a parameter. Then

This theorem was generalized as follows. 


$$
\begin{aligned}
& (b-a) S_{n} \max \left\{\frac{(u-a)^{n}}{n !}, \frac{(b-u)^{n}}{n !}\right\} \\
& +\left[\begin{array}{l}
\frac{(u-a)^{n+1}-(u-b)^{n+1}}{(n+1) !} \\
-(b-a) \max \left\{\frac{(u-a)^{n}}{n !}, \frac{(b-u)^{n}}{n !}\right\}
\end{array}\right] \Gamma \\
& \leq(-1)^{n} \int_{a}^{b} f(t) d t \\
& +\sum_{i=0}^{n-1} \frac{(u-a)^{n+i}-(u-b)^{n+i}}{(n-i) !}(-1)^{i} f^{(n-i-1)}(u) \\
& \leq(b-a) S_{n} \max \left\{\frac{(u-a)^{n}}{n !}, \frac{(b-u)^{n}}{n !}\right\} \\
& +\left[\begin{array}{l}
\frac{(u-a)^{n+1}-(u-b)^{n+1}}{(n+1) !} \\
-(b-a) \max \left\{\frac{(u-a)^{n}}{n !}, \frac{(b-u)^{n}}{n !}\right\}
\end{array}\right] \lambda,
\end{aligned}
$$

where $S_{n}$ is defined by (1.6).

Theorem 1.4 ([[5], Theorem 3]). Let $u \in \mathbb{R}$ and $f(t)$ be $n$ time differentiable on the closed interval $[a, b]$ such that $\gamma \leq f^{(n)}(t) \leq \Gamma$ for $t \in[a, b]$ and $n \in \mathbb{N}$. Then

$$
\begin{aligned}
& {\left[\begin{array}{l}
(b-a) \max \left\{\frac{(u-a)^{n}}{n !}, \frac{(b-u)^{n}}{n !}\right\} \\
+\frac{(b-u)^{n+1}-(a-u)^{n+1}}{(n+1) !}
\end{array}\right] \gamma} \\
& -(b-a) S_{n} \max \left\{\frac{|u-a|^{n}}{n !}, \frac{|b-u|^{n}}{n !}\right\} \\
& \leq(-1)^{n} \int_{a}^{b} f(t) d t \\
& +\sum_{i=0}^{n-1}(-1)^{i} \frac{\left[\begin{array}{l}
(b-u)^{n-i} f^{(n-i-1)}(b) \\
-(a-u)^{n-i} f^{(n-i-1)}(a)
\end{array}\right]}{(n-i) !} \\
& \leq\left[\begin{array}{c}
(b-a) \max \left\{\frac{|u-a|^{n}}{n !}, \frac{|b-u|^{n}}{n !}\right\} \\
+\frac{(b-u)^{n+1}-(a-u)^{n+1}}{(n+1) !}
\end{array}\right] \Gamma \\
& -(b-a) S_{n} \max \left\{\frac{|u-a|^{n}}{n !}, \frac{|b-u|^{n}}{n !}\right\} \text {, }
\end{aligned}
$$

where $S_{n}$ is defined by (1.6).

Theorem 1.5 ([[5], Theorem 5]). Let $\left\{P_{i}(t, x)\right\}_{i=0}^{\infty}$ be a harmonic sequence of polynomials, that is,

$$
P_{i}^{\prime}(t):=\frac{\partial P_{i}(t, x)}{\partial t}=P_{i-1}(t, x):=P_{i-1}(t)
$$

and $P_{0}(t, x)=1$ for all defined $(t, x)$ and $i \in N$. Further let $f(t)$ be $n$-time differentiable on $[a, b]$ such that $\gamma \leq f^{(n)}(t) \leq \Gamma$ for $t \in[a, b]$ and $n \in N$. Then, for any constant $\alpha \in \mathbb{R}$, we have

$$
\begin{aligned}
& {\left[\alpha+\max _{t \in[a, b]}\left|p_{n}(t)+\alpha\right|\right] S_{n}} \\
& -\left(\max _{t \in[a, b]}\left|p_{n}(t)+\alpha\right|+\frac{p_{n+1}(b)-p_{n+1}(a)}{b-a}+\alpha\right) \Gamma \\
& \leq(-1)^{n+1}\left[\frac{1}{b-a} \int_{a}^{b} f(t) \mathrm{d} t\right. \\
& \left.+\sum_{i=1}^{n}(-1)^{i} \frac{p_{i}(b) f^{(i-1)}(b)-p_{i}(a) f^{(i-1)}(a)}{b-a}\right] \\
& \leq\left[\alpha-\max _{t \in[a, b]}\left|p_{n}(t)+\alpha\right|\right] S_{n} \\
& +\left(\max _{t \in[a, b]}\left|p_{n}(t)+\alpha\right|-\frac{p_{n+1}(b)-p_{n+1}(a)}{b-a}-\alpha\right) \Gamma
\end{aligned}
$$

and

$$
\begin{aligned}
& {\left[\alpha-\max _{t \in[a, b]}\left|p_{n}(t)+\alpha\right|\right] S_{n}} \\
& +\left(\max _{t \in[a, b]}\left|p_{n}(t)+\alpha\right|-\frac{p_{n+1}(b)-p_{n+1}(a)}{b-a}-\alpha\right) \gamma \\
& \leq(-1)^{n+1}\left[\frac{1}{b-a} \int_{a}^{b} f(t) \mathrm{d} t\right. \\
& \left.+\sum_{i=1}^{n}(-1)^{i} \frac{p_{i}(b) f^{(i-1)}(b)-p_{i}(a) f^{(i-1)}(a)}{b-a}\right] \\
& \leq\left[\alpha+\max _{t \in[a, b]}\left|p_{n}(t)+\alpha\right|\right] S_{n} \\
& -\left(\max _{t \in[a, b]}\left|p_{n}(t)+\alpha\right|+\frac{p_{n+1}(b)-p_{n+1}(a)}{b-a}+\alpha\right) \gamma .
\end{aligned}
$$

where $S_{n}$ is defined by (1.6).

Theorem 1.6 ([[5], Theorem 7]). Let $\left\{P_{i}(t)\right\}_{i=0}^{\infty}$ and $\left\{Q_{i}(t)\right\}_{i=0}^{\infty}$ be two harmonic sequences of polynomials, $\alpha$ and $\beta$ be two real constants, and $u \in[a, b]$. Further let $f(t)$ be $n$-time differentiable on $[a, b]$ such that $\gamma \leq f^{(n)}(t) \leq \Gamma$ for $t \in[a, b]$ and $n \in \mathrm{N}$. Then

$$
\begin{aligned}
& {\left[\frac{Q_{n+1}(b)-P_{n+1}(a)}{b-a}+\frac{P_{n+1}(u)-Q_{n+1}(u)}{b-a}\right.} \\
& \left.+\frac{(\alpha-\beta) u+(b \beta-a \alpha)}{b-a}+C(u)\right] \gamma-C(u) S_{n} \\
& \leq \frac{(-1)^{n}}{b-a} \int_{a}^{b} f(t) \mathrm{d} t \\
& +\sum_{i=0}^{n-1}(-1)^{n+i} \frac{Q_{i}(b) f^{(i-1)}(b)-P_{i}(a) f^{(i-1)}(a)}{b-a}
\end{aligned}
$$




$$
\begin{aligned}
& +\sum_{i=0}^{n-1}(-1)^{n+i} \frac{P_{i}(u)-Q_{i}(u)}{b-a} f^{(i-1)}(u) \\
& +\frac{\beta f^{(n-1)}(b)-\alpha f^{n}(a)}{b-a}+\frac{(\alpha-\beta) f^{(n-1)}(u)}{b-a} \\
& \leq\left[\frac{Q_{n+1}(b)-P_{n+1}(a)}{b-a}+\frac{P_{n+1}(u)-Q_{n+1}(u)}{b-a}\right. \\
& \left.+\frac{(\alpha-\beta) u+(b \beta-a \alpha)}{b-a}-C(u)\right] \gamma+C(u) S_{n}
\end{aligned}
$$

and

$$
\begin{aligned}
& {\left[\frac{Q_{n+1}(b)-P_{n+1}(a)}{b-a}+\frac{P_{n+1}(u)-Q_{n+1}(u)}{b-a}\right.} \\
& \left.+\frac{(\alpha-\beta) u+(b \beta-a \alpha)}{b-a}-C(u)\right] \Gamma+C(u) S_{n} \\
& \leq \frac{(-1)^{n}}{b-a} \int_{a}^{b} f(t) \mathrm{d} t \\
& +\sum_{i=0}^{n-1}(-1)^{n+i} \frac{Q_{i}(b) f^{(i-1)}(b)-P_{i}(a) f^{(i-1)}(a)}{b-a} \\
& +\sum_{i=0}^{n-1}(-1)^{n+i} \frac{P_{i}(u)-Q_{i}(u)}{b-a} f^{(i-1)}(u) \\
& +\frac{\beta f^{(n-1)}(b)-\alpha f^{n}(a)}{b-a}+\frac{(\alpha-\beta) f^{(n-1)}(u)}{b-a} \\
& \leq\left[\frac{Q_{n+1}(b)-P_{n+1}(a)}{b-a}+\frac{P_{n+1}(u)-Q_{n+1}(u)}{b-a}\right. \\
& \left.+\frac{(\alpha-\beta) u+(b \beta-a \alpha)}{b-a}+C(u)\right] \Gamma-C(u) S_{n},
\end{aligned}
$$

Where $S_{n}$ is defined by (1.6) and

$$
C(u)=\max \left\{\max _{t \in[a, u]}\left|P_{n}(t)+\alpha\right|, \max _{t \in[u, b]}\left|Q_{n}(t)+\beta\right|\right\} .
$$

The aim of this paper is to, by establishing two integral identities and Hölder integral inequality, generalize the above six theorems recited from [5] to more general cases.

\section{Lemmas}

For generalizing the above six theorems recited from [5] to more general cases, we need the following integral identities.

Lemma 2.1. For $n \in \mathrm{N}$, let $f:[a, b] \rightarrow \mathbb{R}$ be a $n$-time differentiable function on $[a, b]$, and let $g_{1}:[a, x] \rightarrow \mathbb{R} \quad$ and $\quad g_{2}:[x, b] \rightarrow \mathbb{R} \quad$ be $n$-time differentiable functions for some $x \in[a, b]$, than

$$
\begin{aligned}
& \int_{a}^{b} g(t) f^{(n)}(t) \mathrm{d} t \\
& =\sum_{i=0}^{n-1}(-1)^{i}\left[\left(g_{1}^{(i)}(x)-g_{2}^{(i)}(x)\right) f^{(n-i-1)}(x)\right. \\
& \left.+\left(g_{2}^{(i)}(b) f^{(n-i-1)}(b)-g_{1}^{(i)}(a) f^{(n-i-1)}(a)\right)\right] \\
& +(-1)^{n} \int_{a}^{b} g^{(n)}(t) f(t) \mathrm{d} t
\end{aligned}
$$

where

$$
\begin{aligned}
& g(t)=\left\{\begin{array}{l}
g_{1}(t), t \in[a, x], \\
g_{2}(t), t \in(x, b] .
\end{array}\right. \\
& g^{(i)}(t)=\left\{\begin{array}{l}
g_{1}^{(i)}(t), t \in[a, x), \\
g_{2}^{(i)}(t), t \in(x, b],
\end{array}\right.
\end{aligned}
$$

and $g^{(i)}\left(x^{-}\right)=g_{1}^{(i)}(x), g^{(i)}\left(x^{+}\right)=g_{2}^{(i)}(x)$ for $1 \leq i \leq n$.

Proof. When $n=1$, it is not difficult to obtain that

$$
\begin{aligned}
& \int_{a}^{b} g(t) f^{\prime}(t) \mathrm{d} t=\left(g_{1}(x)-g_{2}(x)\right) f(x) \\
& +\left(g_{2}(b) f(b)-g_{1}(a) f(a)\right)-\int_{a}^{b} g^{\prime}(t) f(t) \mathrm{d} t .
\end{aligned}
$$

Suppose that the inequality (2.1) holds for $n=k \geq 2$. For $n=k+1$, by integration by parts, we obtain

$$
\begin{aligned}
& \int_{a}^{b} g(t) f^{(k+1)}(t) \mathrm{d} t=\int_{a}^{b} g(t)\left[f^{\prime}(t)\right]^{(k)} \mathrm{d} t \\
& =\sum_{i=0}^{k-1}(-1)^{i}\left[\left(g_{1}^{(i)}(x)-g_{2}^{(i)}(x)\right) f^{(k-i)}(x)\right. \\
& \left.+\left(g_{2}^{(i)}(b) f^{(k-i)}(b)-g_{1}^{(i)}(a) f^{(k-i)}(a)\right)\right] \\
& +(-1)^{k} \int_{a}^{b} g^{(k)}(t) f^{\prime}(t) \mathrm{d} t \\
& =\sum_{i=0}^{k}(-1)^{i}\left[\left(g_{1}^{(i)}(x)-g_{2}^{(i)}(x)\right) f^{(k-i)}(x)\right. \\
& \left.+\left(g_{2}^{(i)}(b) f^{(k-i)}(b)-g_{1}^{(i)}(a) f^{(k-i)}(a)\right)\right] \\
& +(-1)^{k+1} \int_{a}^{b} g^{(k+1)}(t) f(t) \mathrm{d} t .
\end{aligned}
$$

By induction, the proof of inequality (2.1) is complete.

Lemma 2.2 For $n \in \mathrm{N}$, let $f:[a, b] \rightarrow \mathbb{R}$ be a $n$-time differentiable function on $[a, b]$ and, for $x \in[a, b]$ let $g_{1}:[a, x] \rightarrow \mathbb{R}$ and $g_{2}:[x, b] \rightarrow \mathbb{R}$ be $n$-time differentiable functions, then

$$
\begin{aligned}
& \int_{a}^{b} g(t) f^{(n)}(t) \mathrm{d} t \\
& =\sum_{i=0}^{n-1}(-1)^{i}\left[\left(g_{1}^{(i)}(x)-g_{2}^{(i)}(x)\right) f^{(n-i-1)}(x)\right. \\
& \left.+\left(g_{2}^{(i)}(b) f^{(n-i-1)}(b)-g_{1}^{(i)}(a) f^{(n-i-1)}(a)\right)\right] \\
& +(\alpha-\beta) f^{(n-1)}(x)+\left(\beta f^{(n-1)}(b)-\alpha f^{(n-1)}(a)\right) \\
& +(-1)^{n} \int_{a}^{b} g^{(n)}(t) f(t) \mathrm{d} t .
\end{aligned}
$$

where

$$
g(t)=\left\{\begin{array}{l}
g_{1}(t)+\alpha, t \in[a, x] \\
g_{2}(t)+\beta, t \in(x, b]
\end{array}\right.
$$

and $g^{(i)}(t)$ for $1 \leq i \leq n$ are same with (2.2).

\section{Main results}

Now we are in a position to generalize the above six theorems recited from [5] to more general cases. 
Theorem 3.1. For $n \in \mathrm{N}$, let $f:[a, b] \subset \mathbb{R} \rightarrow \mathbb{R}$ be $n$ time differentiable such that $\gamma \leq f^{(n)}(t) \leq \Gamma$ for $t \in[a, b]$. for $x \in[a, b]$ let $g_{1}:[a, x] \rightarrow \mathbb{R} g_{2}:[x, b] \rightarrow \mathbb{R}$ are $n$-time differentiable functions. Then

$$
\begin{aligned}
& (b-a) S_{n} \max \left\{\max _{t \in[a, x]}\left|g_{1}(t)\right|, \max _{t \in[x, b]}\left|g_{2}(t)\right|\right\} \\
& +\left(G(a, b ; g)-(b-a) \max \left\{\begin{array}{c}
\max _{t \in[a, x]}\left|g_{1}(t)\right|, \mid \\
\max _{t \in[x, b]}\left|g_{2}(t)\right|
\end{array}\right\}\right) \Gamma \\
& \leq \sum_{i=0}^{n-1}(-1)^{i}\left[\left(g_{1}^{(i)}(x)-g_{2}^{(i)}(x)\right) f^{(n-i-1)}(x)\right. \\
& \left.+\left(g_{2}^{(i)}(b) f^{(n-i-1)}(b)-g_{1}^{(i)}(a) f^{(n-i-1)}(a)\right)\right] \\
& +(-1)^{n} \int_{a}^{b} g^{(n)}(t) f(t) \mathrm{d} t \\
& \leq(b-a) S_{n} \max \left\{\max _{t \in[a, x]}\left|g_{1}(t)\right|, \max _{t \in[x, b]}\left|g_{2}(t)\right|\right\} \\
& +\left(G(a, b ; g)-(b-a) \max \left\{\begin{array}{c}
\max _{t \in[a, x]}\left|g_{1}(t)\right|, \mid \\
\max _{t \in[x, b]}\left|g_{2}(t)\right|
\end{array}\right\} \gamma,\right.
\end{aligned}
$$

where $S_{n}$ is defined by (1.6), $g(t)$ and $g^{(i)}(t)$ are defined as in (2.2) and

$$
G(\mathrm{a}, \mathrm{b} ; \mathrm{g})=\frac{1}{b-a} \int_{a}^{b} g(t) \mathrm{d} t .
$$

Proof. By Lemma 2.1, we have

$$
\begin{aligned}
& \int_{a}^{b} g(t)\left[f^{(n)}(t)-\gamma\right] \mathrm{d} t \\
& =(-1)^{n} \int_{a}^{b} g^{(n)}(t) f(t) \mathrm{d} t-\gamma(b-a) G(a, b ; g) \\
& +\sum_{i=0}^{n-1}(-1)^{i}\left[\left(g_{1}^{(i)}(x)-g_{2}^{(i)}(x)\right) f^{(n-i-1)}(x)\right. \\
& \left.+\left(g_{2}^{(i)}(b) f^{(n-i-1)}(b)-g_{1}^{(i)}(a) f^{(n-i-1)}(a)\right)\right]
\end{aligned}
$$

and

$$
\begin{aligned}
& \int_{a}^{b} g(t)\left[\Gamma-f^{(n)}(t)\right] \mathrm{d} t \\
& =\Gamma(b-a) G(a, b ; g)-(-1)^{n} \int_{a}^{b} g^{(n)}(t) f(t) \mathrm{d} t \\
& -\sum_{i=0}^{n-1}(-1)^{i}\left[\left(g_{1}^{(i)}(x)-g_{2}^{(i)}(x)\right) f^{(n-i-1)}(x)\right. \\
& \left.+\left(g_{2}^{(i)}(b) f^{(n-i-1)}(b)-g_{1}^{(i)}(a) f^{(n-i-1)}(a)\right)\right] .
\end{aligned}
$$

On the other hand, by the Hölder inequality,

$$
\begin{aligned}
& \int_{a}^{b} g(t)\left[f^{(n)}(t)-\gamma\right] \mathrm{d} t \leq \int_{a}^{b}|g(t)|\left|f^{(n)}(t)-\gamma\right| \mathrm{d} t \\
& \leq \max _{t \in[a, b]}|g(t)| \int_{a}^{b}\left[f^{(n)}(t)-\gamma\right] \mathrm{d} t \\
& =(b-a)\left(S_{n}-\gamma\right) \max \left\{\max _{t \in[a, x]}\left|g_{1}(t)\right|, \max _{t \in[x, b]}\left|g_{2}(t)\right|\right\}
\end{aligned}
$$

and

$$
\begin{aligned}
& \int_{a}^{b} g(t)\left[\Gamma-f^{(n)}(t)\right] \mathrm{d} t \leq \int_{a}^{b}|g(t)|\left|\Gamma-f^{(n)}(t)\right| \mathrm{d} t \\
& \leq \max _{t \in[a, b]}|g(t)| \int_{a}^{b}\left[\Gamma-f^{(n)}(t)\right] \mathrm{d} t \\
& =(b-a)\left(\Gamma-S_{n}\right) \max \left\{\max _{t \in[a, x]}\left|g_{1}(t)\right|, \max _{t \in[x, b]}\left|g_{2}(t)\right|\right\} .
\end{aligned}
$$

Combining (3.3) to (3.6) yields (3.1). Theorem 3.1 is thus proved.

Remark 1. From taking

$$
g(t)=\left\{\begin{array}{l}
\frac{(t-a)^{n}}{n !}, t \in[a, x], \\
\frac{(t-b)^{n}}{n !}, t \in(x, b]
\end{array}\right.
$$

in (3.1), the double inequality (1.7) followes.

If taking $x=b, g_{2}(t)=0$ in Theorem 3.1, we can derive the following corollary.

Corollary 3.1.1. For $n \in \mathrm{N}$, let $f:[a, b] \rightarrow \mathbb{R}$ be $n-$ time differentiable such that $\gamma \leq f^{(n)}(t) \leq \Gamma$ for $t \in[a, b]$ and let $g:[a, b] \rightarrow \mathbb{R}$ be $n$-time differentiable. Then

$$
\begin{aligned}
& (b-a) S_{n} \max _{t \in[a, b]}|g(t)| \\
& +(b-a)\left[G(a, b ; g)-\max _{t \in[a, b]}|g(t)|\right] \Gamma \\
& \leq(-1)^{n} \int_{a}^{b} g^{(n)}(t) f(t) \mathrm{d} t \\
& +\sum_{i=0}^{n-1}(-1)^{i}\left[g^{(i)}(b) f^{(n-i-1)}(b)-g^{(i)}(a) f^{(n-i-1)}(a)\right] \\
& \leq(b-a) S_{n} \max _{t \in[a, b]}|g(t)| \\
& +(b-a)\left[G(a, b ; g)-\max _{t \in[a, b]}|g(t)|\right] \gamma .
\end{aligned}
$$

Proof. This follow from putting $x=b, g(t)=g_{1}(t)$, and $g_{2}(t)=0$ in Theorem 3.1.

Remark 2. If letting $g(t)=\frac{(t-u)^{n}}{n !}$ for $u \in \mathbb{R}$ in (3.7), the double inequality (1.8) may be derived.

Corollary 3.1.2. Under the conditions of Theorem 3.1, if $x=\frac{a+b}{2}$, then

$$
\begin{aligned}
& (b-a)\left[S_{n} \max \left\{\max _{t \in[a, x]}\left|g_{1}(t)\right|, \max _{t \in[x, b]}\left|g_{2}(t)\right|\right\}\right. \\
& \left.+\left(G(a, b ; g)-\max \left\{\max _{t \in[a, x]}\left|g_{1}(t)\right|, \max _{t \in[x, b]}\left|g_{2}(t)\right|\right\}\right) \Gamma\right] \\
& \leq \sum_{i=0}^{n-1}(-1)^{i}\left[\left(g_{1}^{(i)}\left(\frac{a+b}{2}\right)-g_{2}^{(i)}\left(\frac{a+b}{2}\right)\right) f^{(n-i-1)}\left(\frac{a+b}{2}\right)\right. \\
& \left.+\left(g_{2}^{(i)}(b) f^{(n-i-1)}(b)-g_{1}^{(i)}(a) f^{(n-i-1)}(a)\right)\right]
\end{aligned}
$$


$+(-1)^{n} \int_{a}^{b} g^{(n)}(t) f(t) \mathrm{d} t$

$\leq(b-a)\left[S_{n} \max \left\{\max _{t \in[a, x]}\left|g_{1}(t)\right|, \max _{t \in[x, b]}\left|g_{2}(t)\right|\right\}\right.$

$\left.+\left(G(a, b ; g)-\max \left\{\max _{t \in[a, x]}\left|g_{1}(t)\right|, \max _{t \in[x, b]}\left|g_{2}(t)\right|\right\}\right) \gamma\right]$.

Corollary 3.1.3. Under the conditions of Theorem 3.1, if $n=2$, then

$$
\begin{aligned}
& (b-a)\left[S_{2} \max \left\{\max _{t \in[a, x]}\left|g_{1}(t)\right|, \max _{t \in[x, b]}\left|g_{2}(t)\right|\right\}\right. \\
& \left.+\left(G(a, b ; g)-\max \left\{\max _{t \in[a, x]}\left|g_{1}(t)\right|, \max _{t \in[x, b]}\left|g_{2}(t)\right|\right\}\right) \Gamma\right] \\
& \leq \sum_{i=0}^{1}(-1)^{i}\left[\left(g_{1}^{(i)}(x)-g_{2}^{(i)}(x)\right) f^{(1-i)}(x)\right. \\
& \left.+g_{2}^{(i)}(b) f^{(1-i)}(b)-g_{1}^{(i)}(a) f^{(1-i)}(a)\right] \\
& +\int_{a}^{b} g^{\prime \prime}(t) f(t) \mathrm{d} t \\
& \leq(b-a)\left[S_{2} \max \left\{\max _{t \in[a, x]}\left|g_{1}(t)\right|, \max _{t \in[x, b]}\left|g_{2}(t)\right|\right\}\right. \\
& \left.+\left(G(a, b ; g)-\max \left\{\max _{t \in[a, x]}\left|g_{1}(t)\right|, \max _{t \in[x, b]}\left|g_{2}(t)\right|\right\}\right) \gamma\right]
\end{aligned}
$$

Theorem 3.2. For $n \in \mathrm{N}$, let $f:[a, b] \rightarrow \mathbb{R}$ be a $n$-time differentiable function on $[a, b]$ and, for $x \in[a, b]$ let $g_{1}:[a, x] \rightarrow \mathbb{R} \quad$ and $\quad g_{2}:[x, b] \rightarrow \mathbb{R} \quad$ be $n$-time differentiable functions. Then, for $\alpha, \beta$ being real constants,

$$
\begin{aligned}
& {[G(a, b ; g)+H(x)] \gamma-H(x) S_{n}} \\
& \leq \frac{(-1)^{n}}{b-a} \int_{a}^{b} g^{(n)}(t) f(t) \mathrm{d} t \\
& +\frac{(\alpha-\beta) f^{(n-1)}(x)+\beta f^{(n-1)}(b)-\alpha f^{(n-1)}(a)}{b-a} \\
& +\sum_{i=0}^{n-1} \frac{(-1)^{i}}{b-a}\left[\left(g_{1}^{(i)}(x)-g_{2}^{(i)}(x)\right) f^{(n-i-1)}(x)\right. \\
& \left.+g_{2}^{(i)}(b) f^{(n-i-1)}(b)-g_{1}^{(i)}(a) f^{(n-i-1)}(a)\right] \\
& \leq[G(a, b ; g)-H(x)] \gamma+H(x) S_{n}
\end{aligned}
$$

and

$$
\begin{aligned}
& {[G(a, b ; g)-H(x)] \Gamma+H(x) S_{n}} \\
& \leq \frac{(-1)^{n}}{b-a} \int_{a}^{b} g^{(n)}(t) f(t) \mathrm{d} t \\
& +\frac{(\alpha-\beta) f^{(n-1)}(x)+\beta f^{(n-1)}(b)-\alpha f^{(n-1)}(a)}{b-a} \\
& +\sum_{i=0}^{n-1} \frac{(-1)^{i}}{b-a}\left[\left(g_{1}^{(i)}(x)-g_{2}^{(i)}(x)\right) f^{(n-i-1)}(x)\right. \\
& \left.+g_{2}^{(i)}(b) f^{(n-i-1)}(b)-g_{1}^{(i)}(a) f^{(n-i-1)}(a)\right] \\
& \leq[G(a, b ; g)+H(x)] \Gamma-H(x) S_{n},
\end{aligned}
$$

where $S_{n}, G(a, b ; g), g(t)$ and $G^{(i)}(t)$ are defined respectively by (1.6), (3.2), (2.4), (2.2) and

$$
H(x)=\max \left\{\max _{t \in[a, x]}\left|g_{1}(t)+\alpha\right|, \max _{t \in[x, b]}\left|g_{2}(t)+\beta\right|\right\} .
$$

Proof. Applying Lemma 2.2 results in

$$
\begin{aligned}
& \int_{a}^{b} g(t)\left[f^{(n)}(t)-\gamma\right] \mathrm{d} t \\
& =(-1)^{n} \int_{a}^{b} g^{(n)}(t) f(t) \mathrm{dt}-(b-a) \gamma G(a, b ; g) \\
& +(\alpha-\beta) f^{(n-1)}(x)+\left(\beta f^{(n-1)}(b)-\alpha f^{(n-1)}(a)\right) \\
& +\sum_{i=0}^{n-1}(-1)^{i}\left[\left(g_{1}^{(i)}(x)-g_{2}^{(i)}(x)\right) f^{(n-i-1)}(x)\right. \\
& \left.+\left(g_{2}^{(i)}(b) f^{(n-i-1)}(b)-g_{1}^{(i)}(a) f^{(n-i-1)}(a)\right)\right]
\end{aligned}
$$

and

$$
\begin{aligned}
& \int_{a}^{b} g(t)\left[\Gamma-f^{(n)}(t)\right] \mathrm{d} t \\
& =(-1)^{n+1} \int_{a}^{b} g^{(n)}(t) f(t) \mathrm{d} t+(b-a) \Gamma G(a, b ; g) \\
& -(\alpha-\beta) f^{(n-1)}(x)-\left(\beta f^{(n-1)}(b)-\alpha f^{(n-1)}(a)\right) \\
& -\sum_{i=0}^{n-1}(-1)^{i}\left[\left(g_{1}^{(i)}(x)-g_{2}^{(i)}(x)\right) f^{(n-i-1)}(x)\right. \\
& \left.+\left(g_{2}^{(i)}(b) f^{(n-i-1)}(b)-g_{1}^{(i)}(a) f^{(n-i-1)}(a)\right)\right] .
\end{aligned}
$$

It is easy to show, by the Hölder inequality, that

$$
\begin{aligned}
& \left|\int_{a}^{b} g(t)\left[f^{(n)}(t)-\gamma\right] \mathrm{d} t\right| \\
& \leq \int_{a}^{b}|g(t)|\left|f^{(n)}(t)-\gamma\right| \mathrm{d} t \\
& \leq \max _{t \in[a, b]}|g(t)| \int_{a}^{b}\left[f^{(n)}(t)-\gamma\right] \mathrm{d} t \\
& =(b-a)\left(S_{n}-\gamma\right) H(x)
\end{aligned}
$$

and

$$
\begin{aligned}
& \left|\int_{a}^{b} g(t)\left[\Gamma-f^{(n)}(t)\right] \mathrm{d} t\right| \\
& \leq \int_{a}^{b}|g(t)|\left|\Gamma-f^{(n)}(t)\right| \mathrm{d} t \\
& \leq \max _{t \in[a, b]}|g(t)| \int_{a}^{b}\left[f^{(n)}(t)-\gamma\right] \mathrm{d} t \\
& =(b-a)\left(\Gamma-S_{n}\right) H(x) .
\end{aligned}
$$

Combining the above identitie and inequalities yields Theorem 3.2.

Remark 3.3. For $\alpha, \beta \in \mathbb{R}$, setting

$$
g(t)=\left\{\begin{array}{l}
P_{n}(t)+\alpha, t \in[a, x], \\
Q_{n}(t)+\beta, t \in(x, b]
\end{array}\right.
$$

in Theorem 3.2, where $\left\{P_{i}(t)\right\}_{i=0}^{\infty}$ and $\left\{Q_{i}(t)\right\}_{i=0}^{\infty}$ are two harmonic sequences of polynomials, reveals the double inequalities (1.12) and (1.13).

Corollary 3.1.1. Let $f:[a, b] \subset \mathbb{R} \rightarrow \mathbb{R}$ be $n$-time differentiable on the closed interval $[a, b]$ such that 
$\gamma \leq f^{(n)}(t) \leq \Gamma$ for $t \in[a, b], x \in[a, b]$ and $g:[a, b] \rightarrow \mathbb{R}$ be $n$-time differentiable function for $n \in N$, let $\alpha, \beta$ be a real constant. Then

$[G(a, b ; g)+H(x)] \gamma-H(x) S_{n}$

$\leq \frac{(-1)^{n}}{b-a} \int_{a}^{b} g^{(n)}(t) f(t) \mathrm{d} t+\frac{\alpha\left(f^{(n-1)}(b)-f^{(n-1)}(a)\right)}{b-a}$

$+\sum_{i=0}^{n-1}(-1)^{i} \frac{\left.g^{(i)}(b) f^{(n-i-1)}(b)-g^{(i)}(a) f^{(n-i-1)}(a)\right)}{b-a}$

$\leq[G(a, b ; g)-H(x)] \gamma+H(x) S_{n}$

and

$[G(a, b ; g)-H(x)] \Gamma+H(x) S_{n}$

$\leq \frac{(-1)^{n}}{b-a} \int_{a}^{b} g^{(n)}(t) f(t) \mathrm{d} t+\frac{\alpha\left(f^{(n-1)}(b)-f^{(n-1)}(a)\right)}{b-a}$

$+\sum_{i=0}^{n-1}(-1)^{i} \frac{\left.g(i)(b) f^{(n-i-1)}(b)-g^{(i)}(a) f^{(n-i-1)}(a)\right)}{b-a}$

$\leq[G(a, b ; g)+H(x)] \Gamma-H(x) S_{n}$.

Proof. This follows from taking $x=b$, $g(t)=g_{1}(t), g_{2}(t)=0$ and $\alpha=\beta$ in Theorem 3.2.

Remark 3.4. Taking $g(t)=P_{n}(t)+\alpha$ in (3.13) and (3.14), $\left\{P_{i}(t)\right\}_{i=0}^{\infty}$ be a harmonic of polynomials may derive the double inequalities (1.10) and (1.11).

Corollary 3.2.2. Under the conditions of Theorem 3.2, we have

$$
\begin{aligned}
& {\left[G(a, b ; g)+H\left(\frac{a+b}{2}\right)\right] \gamma-H\left(\frac{a+b}{2}\right) S_{n}} \\
& \leq \frac{(-1)^{n}}{b-a} \int_{a}^{b} g^{(n)}(t) f(t) \mathrm{d} t+\frac{(\alpha-\beta) f^{(n-1)}\left(\frac{a+b}{2}\right)}{b-a} \\
& +\frac{\left(\beta f^{(n-1)}(b)-\alpha f^{(n-1)}(a)\right)}{b-a} \\
& +\sum_{i=0}^{n-1} \frac{(-1)^{i}}{b-a}\left[\left(g_{1}^{(i)}\left(\frac{a+b}{2}\right)-g_{2}^{(i)}\left(\frac{a+b}{2}\right)\right) f^{(n-i-1)}(x)\right. \\
& \left.+g_{2}^{(i)}(b) f^{(n-i-1)}(b)-g_{1}^{(i)}(a) f^{(n-i-1)}(a)\right] \\
& \leq\left[G(a, b ; g)-H\left(\frac{a+b}{2}\right)\right] \gamma+H\left(\frac{a+b}{2}\right) S_{n}
\end{aligned}
$$

and

$$
\begin{aligned}
& {\left[G(a, b ; g)-H\left(\frac{a+b}{2}\right)\right] \Gamma+H\left(\frac{a+b}{2}\right) S_{n}} \\
& \leq \frac{(-1)^{n}}{b-a} \int_{a}^{b} g^{(n)}(t) f(t) \mathrm{d} t+\frac{(\alpha-\beta) f^{(n-1)}\left(\frac{a+b}{2}\right)}{b-a} \\
& +\frac{\beta f^{(n-1)}(b)-\alpha f^{(n-1)}(a)}{b-a} \\
& +\sum_{i=0}^{n-1} \frac{(-1)^{i}}{b-a}\left[\left(g_{1}^{(i)}\left(\frac{a+b}{2}\right)-g_{2}^{(i)}\left(\frac{a+b}{2}\right)\right) f^{(n-i-1)}\left(\frac{a+b}{2}\right)\right. \\
& \left.+\left(g_{2}^{(i)}(b) f^{(n-i-1)}(b)-g_{1}^{(i)}(a) f^{(n-i-1)}(a)\right)\right] \\
& \leq\left[G(a, b ; g)+H\left(\frac{a+b}{2}\right)\right] \Gamma-H\left(\frac{a+b}{2}\right) S_{n} .
\end{aligned}
$$

Proof. This follows from putting $x=\frac{a+b}{2}$ in Theorem 3.2.
Corollary 3.2.3. Under the conditions of Theorem 3.2, if $n=2$, then

$$
\begin{aligned}
& {[G(a, b ; g)+H(x)] \gamma-H(x) S_{2}} \\
& \leq \frac{1}{b-a} \int_{a}^{b} g^{\prime \prime}(t) f(t) \mathrm{d} t \\
& +\frac{(\alpha-\beta) f^{\prime}(x)}{b-a}+\frac{\beta f^{\prime}(b)-\alpha f^{\prime}(a)}{b-a} \\
& +\sum_{i=0}^{1} \frac{(-1)^{i}}{b-a}\left[\left(g_{1}^{(i)}(x)-g_{2}^{(i)}(x)\right) f^{(1-i)}(x)\right. \\
& \left.+g_{2}^{(i)}(b) f^{(1-i)}(b)-g_{1}^{(i)}(a) f^{(1-i)}(a)\right] \\
& \leq[G(a, b ; g)-H(x)] \gamma+H(x) S_{2}
\end{aligned}
$$

and

$$
\begin{aligned}
& {[G(a, b ; g)-H(x)] \Gamma+H(x) S_{2}} \\
& \leq \frac{1}{b-a} \int_{a}^{b} g^{\prime \prime}(t) f(t) \mathrm{d} t \\
& +\frac{(\alpha-\beta) f^{\prime}(x)}{b-a}+\frac{\beta f^{\prime}(b)-\alpha f^{\prime}(a)}{b-a} \\
& +\sum_{i=0}^{1} \frac{(-1)^{i}}{b-a}\left[\left(g_{1}^{(i)}(x)-g_{2}^{(i)}(x)\right) f^{(1-i)}(x)\right. \\
& \left.+g_{2}^{(i)}(b) f^{(1-i)}(b)-g_{1}^{(i)}(a) f^{(1-i)}(a)\right] \\
& \leq[G(a, b ; g)+H(x)] \Gamma-H(x) S_{2} .
\end{aligned}
$$

\section{Acknowledgements}

This work was partially supported by the National Natural Science Foundation of China underGrant No. 11361038, China and by the Foundationof the Research Program of Science and Technology at Universities of Inner Mongolia Autonomous Region under Grant No. NJZY14191, China.

\section{References}

[1] R.-F. Bai, F. Qi, and B.-Y. Xi, Hermite-Hadamard type inequalities for the $m$ - and $(\alpha, m)$-logarithmically convex functions, Filomat 27(2013), no. 1, 1-7.

[2] P. Cerone and S. S. Dragomir, Midpoint-type rules from an inequality point of view, Handbook of Analytic-computational Methods in Applied Mathematics, 135-200, Chapman \& Hall/CRC, Boca Raton, FL, 2000.

[3] S. S. Dragomir and C. E. M. Pearce, Selected Topics on HermiteHadamard Type Inequalities and Applications, RGMIA Monographs, Victoria University, 2000; Available online at http://rgmia.org/monographs/hermite_hadamard.html.

[4] C. P. Niculescu and L.-E. Persson, Convex Functions and their Applications, CMS Books in Mathematics/Ouvrages de Mathématiques de la SMC, 23. Springer, New York, 2006.

[5] F. Qi, Z.-L. Wei, and Q. Yang, Generalizations and refinements of Hermite-Hadamard's inequality, Rocky Mountain J. Math. 35 (2005), no. 1, 235-251.

[6] N. Ujević, Some double integral inequalities and applications, Acta Math. Univ. Comenian. (N.S.) 71 (2002), no. 2, 189-199.

[7] B.-Y. Xi and F. Qi, Some Hermite-Hadamard type inequalities for differentiable convex functions and applica-tions, Hacet. J. Math. Stat. 42 (2013), no. 3, 243-257. 